\title{
Aplicativos móveis para praticar a leitura social: análise e avaliação de recursos úteis
}

\author{
Taísa Dantas \\ CAPES. Coordenação de aperfeiçoamento de pessoal de nível superior, Brasil \\ Universidad de Salamanca, España \\ taisadantasbr@gmail.com
}

Cita sugerida: Dantas , T. (2018). Aplicativos móveis para praticar a leitura social: análise e avaliação de recursos úteis. Palabra Clave (La Plata), 7(2), e048. https://doi.org/10.24215/18539912e048

Recibido: 20 de novembro de 2017 - Aceptado: 24 de novembro de 2017 - Publicado: 27 de abril de 2018

(c) (1) (2) Esta obra está bajo licencia Creative Commons Atribución-NoComercial-CompartirIgual 4.0 Internacional

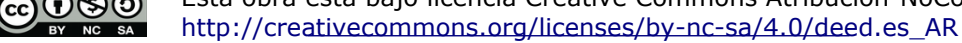




\section{Aplicativos móveis para praticar a leitura social: análise e avaliação de recursos úteis}

Mobile applications to practice social reading: analysis and evaluation of useful tools

Taisa Dantas

CAPES. Coordenação de aperfeiçoamento de pessoal de

nivel superior, Brasil

Universidad de Salamanca, España

taisadantasbr@gmail.com

\section{Resumo:}

Este artigo tem como objetivo compreender como os aplicativos móveis podem ser utilizados para auxiliar os leitores durante a prática da leitura social, a partir da análise dos diferentes recursos disponíveis, dos seus benefícios e limitações. Foi selecionada uma metodologia com abordagem qualitativa de caráter descritivo e analítico, baseada em diferentes estudos enfocados na avaliação de recursos eletrônicos. Neste caso, foram avaliados dez aplicativos de leitura disponíveis nos sistemas operativos Android e iOS, desenvolvidos tanto para a leitura em tablet como para smartphone. Concluiu-se que aplicativos móveis são softwares eficientes para incentivar o leitor a realizar atividades previstas dentro da modalidade da leitura social, principalmente no que corresponde à prática da leitura ativa. Por outro lado, verificou-se que, com relação ao compartilhamento da leitura, até o momento, a maior parte dos aplicativos avaliados apresentam debilidades, principalmente, para incentivar o leitor a interagir com outros leitores, limitandose ao compartilhamento em espaços externos ao aplicativo e, na sua maioria, plataformas não especializadas no âmbito do livro.

Palavras-chave: Leitura social, Aplicativos de leitura, Livros eletrônicos, Promoção de hábitos leitores.

\section{Abstract:}

This article aims to understand how mobile applications can be used to assist readers during social reading practice, through the analysis of different available resources, their benefits, and limitations. This study has a descriptive and analytical qualitative methodology approach, based on studies focused on the evaluation of electronic resources. In this case, ten reading applications available on Android and iOS operating systems designed for both tablet and smartphone reading, were evaluated. Was possible to conclude that mobile applications are efficient software to encourage the reader to carry out activities planned within the modality of social reading. Especially in what corresponds to the practice of active reading. On the other hand, was verified that for sharing the reading practice, until now, most of the evaluated applications hold weaknesses. Mainly, to encourage the reader to interact with other readers. The tools are limited to sharing through non-specialized platforms within the context of the book and outside of the application.

KEYWORDS: Social reading, Reading application, Electronic books, Social reading, Reading habits promotion.

\section{INTRODUÇÃO}

Em 1455, o alemão Johannes Gutenberg apresentou ao mundo o primeiro livro impresso por meio do uso de uma prensa móvel, e o que veio a ser o mais importante incunábulo da história, a tradução em latim da Bíblia, conhecida como a Biblia de Gutenberg (Library of Congress, 2000). Em 1971, o americano Michel Hart digitalizou a Declaração de Independência dos Estados Unidos, criando o primeiro livro eletrônico (Lebert, 2008), isto é o e-incunábulo com maior relevância da história.

Foram necessários cinco séculos para que o livro, no formato como foi criado por Gutenberg, passasse a sofrer modificações realmente significativas. Entretanto, não foi necessário muito mais do que quarenta anos para que o livro, como foi desenvolvido por Hart, em formato eletrônico, se transformasse completamente.

De páginas estáticas, sem possibilidades de realizar marcações ao longo do texto e passível de ser enviado a apenas 100 outros leitores, os livros eletrônicos atualmente são editados de diferentes formas, podem ser interativos e abertos à inserção de informações por parte do leitor, acessível a partir de diferentes fontes e 
distribuídos por meio de páginas web, emails, códigos QR, etc. O livro eletrônico atual é completamente diferente da e-incunábulo de Hart, entretanto, ambos possuem um importante ponto em comum: a necessidade de um software específico para que possa ser lido utilizando um dispositivo eletrônico.

Dentro do contexto da leitura digital, a escolha por qual dispositivo utilizar não é uma tarefa indiferente. A experiência de leitura em um computador não é necessariamente a mesma que em um tablet, assim como, não é igual ler um e-reader ou ler em um smartphone. Por esta razão, dentro do âmbito da pesquisa sobre a leitura, existem diferentes experimentos que buscam compreender qual é o impacto que o uso de cada um destes dispositivos pode gerar no processo e na prática da leitura (Dantas, 2017; Grzeschik, Kruppa, Marti, \& Donner, 2011; Kang \& Eune, 2012; Mangen, 2008; Richardson Jr \& Mahmood, 2012; Tveit \& Mangen, 2014). Igualmente, em virtude das diferentes opções e da importância que podem representar cada uma delas, nos últimos anos começa a aumentar o interesse por parte dos pesquisadores em realizar experiências enfocadas na observação da prática da leitura a partir do uso de diferentes softwares,e mais especificamente de aplicativos móveis utilizados para a leitura (Aamri, Greuter, \& Walz, 2015; Li \& Wu, 2017), como é o caso da pesquisa apresentada neste artigo.

Examinando o cenário no qual é realizada a leitura digital, desde os dispositivos até os aplicativos, compreendemos que a prática da leitura torna-se mais dinâmica, com a melhoria de algumas atividades que já eram realizadas dentro do contexto impresso e com o estímulo para serem realizadas ações que tornaram-se muito mais intensas a partir do crescimento do uso das novas tecnologias da informação no cotidiano, como o ato de compartilhar as atividades desempenhadas, as opiniões sobre determinados argumentos, entre outros.

Verifica-se que ganha destaque o reconhecimento da leitura não somente como uma prática solitária e linear, mas também como uma atividade passível de ser realizada de forma hipertextual e compartilhada, mediante a que denominamos como leitura social.

Neste sentido, apresentamos a continuação uma pesquisa realizada com o objetivo de compreender os benefícios ou inconvenientes que podem ser gerados pelo uso dos aplicativos de leitura digital para a prática da leitura social. Para tanto se analisa, avalia e compara uma série de aplicativos previamente selecionados com o intuito de examinar os seus principais recursos e como estes podem ser utilizados na prática desta modalidade de leitura.

\section{MARCo TEÓRICo}

A leitura social é uma modalidade de leitura que possui diferentes acepções. Pode ser definida como uma prática de leitura em ambientes virtuais na qual o leitor interage com diferentes sistemas dentro e fora do livro (Cordón García, Alonso Arévalo, Gómez Díaz, \& Linder, 2013; Orosa \& García, 2016; Romero, 2012), bem como uma prática que prevê o compartilhamento e a conversa ao redor do livro utilizando plataformas digitais (Rowberry, 2016; Vlieghe, Muls, \& Rutten, 2016). Em ambos os casos, trata-se da prática da leitura com características que fazem referência à leitura ativa è̀ leitura compartilhada, entretanto, com o diferencial de que o leitor passa a utilizar como suporte principal instrumentos vinculados às novas tecnologias (Dantas, 2017).

A leitura ativa é um método no qual o leitor interage com o texto com a intenção de melhorar a sua compreensão (Adler \& Doren, 2001; Palilonis \& Bolchini, 2015; Pearson, Buchanan, Thimbleby, \& Jones, 2012). Esta interação ocorre por meio de intervenções feitas ao longo do texto, como sublinhar, inserir comentários, criar esquemas, resumos, etiquetar, entre outros. Enquanto isto, a leitura compartilhada é uma atividade realizada pelo leitor, habitualmente em uma fase posterior ao ato de ler na qual se comenta o que foi lido,discutindo, fazendo comentários ou críticas.

Levando em consideração todas as características anteriormente destacadas, pode-se definir a leitura social como uma prática da leitura em suportes digitais na qual o leitor tem a possibilidade de compartilhar as 
intervenções que realiza sobre o texto (leitura ativa) com outros leitores e até mesmo o autor do texto (leitura compartilhada), durante ou depois da leitura.

O sentido de socialização, neste contexto, não ocorre somente por meio do compartilhamento de partes dos livros ou comentários em plataformas de redes sociais, mas sim, corresponde a todos os níveis de interação que o leitor pode realizar com a obra, com textos adicionais e com outros leitores. Auxiliados por instrumentos e pelos sistemas que se incorporam ao livro. Estes sistemas podem ser de leitura, recomendação, aquisição ou compartilhamento. Entre os diferentes instrumentos que podem ser utilizados para auxiliar esta prática destacamos os aplicativos móveis utilizados para a leitura digital.

Existem diferentes aplicativos que podem ser utilizados para a leitura de um livro eletrônico, a decisão por um ou por outro ocorre, principalmente, em virtude do formato em que foi disponibilizado (por exemplo epub, mobi, pdf, etc.), ou mesmo impulsionada pelo suporte utilizado ou pela livraria ou biblioteca pela qual foi acessado o livro a ser lido.

O software selecionado para servir como base para a leitura digital pode afetar a compreensão tanto quanto uma má impressão de um livro.Impedindo que o leitor realize atividades básicas como sublinhar uma parte do texto, ou atividades mais específicas como compartilhar um comentário com outros leitores. Por esta razão se torna imprescindível compreender como estão organizados os aplicativos de leitura e como os recursos disponíveis podem auxiliar ao leitor a praticar de forma eficiente a leitura social.

\section{Metodologia}

Este estudo possui uma abordagem qualitativa de caráter descritivo e analítico. Com o intuito de alcançar os objetivos delimitados utilizou-se como fundamentação teórico-metodológica o estudo realizado por Codina (2000) e os realizados por Gómez-Diaz (2012) e Patón-Rodríguez e Goméz-Díaz (2016). O primeiro foi selecionado por sua eficiência em estabelecer uma metodologia de avaliação de recursos digitais (Codina, 2000), enquanto que os seguintes foram selecionados por suas adaptações aos contextos da leitura em ambientes digitais, avaliando diferentes recursos, aplicativos para dispositivos móveis (Gómez-Díaz, 2012) e aplicativos para a leitura em páginas web (Patón-Rodríguez \& Gómez-Díaz, 2016).

A partir do aprofundamento nestes estudos estabeleceu-se para esta pesquisa uma série de açóes a serem desempenhadas. Sendo elas:

- Seleção dos aplicativos a serem avaliados

- Desenvolvimento de planilhas de avaliação

- Definição de critérios, parâmetros e indicadores

- Análise dos aplicativos selecionados

- Avaliação dos benefícios e limites do uso de aplicativos para a prática da leitura social.

\subsection{SELEÇÃo DE APLICATIVOS}

Foram selecionados dez aplicativos (figura 1) indicados entre os mais utilizados de duas lojas virtuais relacionadas ao sistema operativo Android (Google Play Store) e ao sistema operativo iOS (Apple Store). São eles: Wattpad, Kindle, eReader Prestigio, Aldiko, Google Play Books, Kobo, eBoox, iBooks, Bluefire Reader, e Moon+ Reader. 
Estes aplicativos possuem diferentes origens e características, o que torna possível uma compreensão mais exaustiva tanto do ecossistema deste tipo de software, como do seu uso para finalidades especificas, como é o caso da utilização para a prática da leitura social.

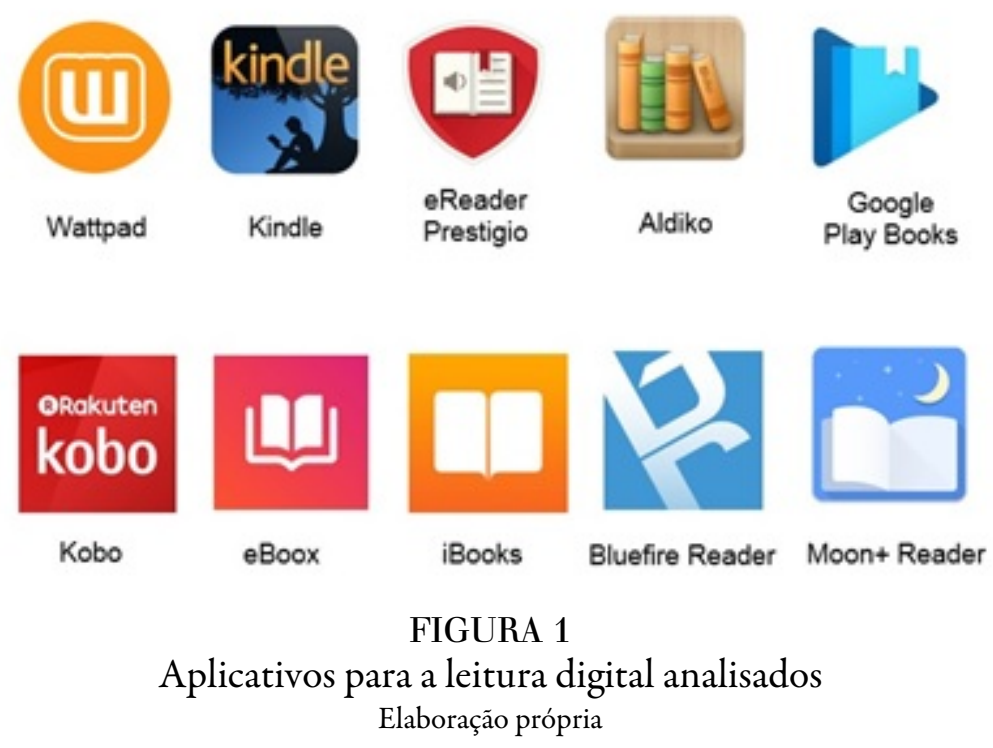

\subsection{Desenvolvimento de Planilha de aValiação}

Embora existam diferentes estudos com características similares a este, ou seja, direcionados à avaliação de recursos eletrônicos (Guillén Ríos \& Ayuso García, 2014; Sam-Anlas \& Stable-Rodríguez, 2016), inclusive enfocados no uso de aplicativos (García-Rodríguez \& Gómez-Díaz, 2015; Gómez Díaz, García Rodríguez, \& Cordón García, 2015; Pilcher, 2016; Schrock, 2016). Com relação ao aspecto delimitado nesta pesquisa nenhum estudo similar foi localizado, e por esta razão foram desenvolvidas planilhas de avaliação exclusivamente considerando a perspectiva analisada, o auxilio à prática da leitura social.

\subsubsection{DEFINIÇÃO DOS CRITÉRIOS, PARÂMETROS E INDICADORES}

Os critérios, parâmetros e indicadores foram definidos a partir dos estudos realizados por Dantas (2017), vinculados à definição de leitura social. A partir desta base teórica foram definidas planilhas de avaliação, nas quais estão incluídos diferentes critérios relevantes a serem considerados para a prática desta modalidade, sendo eles: interação com o texto, interação com outros sistemas, e interação com outros leitores.

\subsubsection{INTERAÇÃO COM O TEXTO}

A primeira planilha (tabela 1) é composta por dois parâmetros: paratexto editorial e leitura ativa. O primeiro corresponde a avaliação de recursos que proporcionam ao leitor informações que facilitem uma maior compreensão do tipo de material que está sendo lido, ou seja, informações sobre o autor e sobre a sua obra. Sendo assim, é composto por indicadores como a presença do titulo, autor, editora, ano, biografia, e bibliografia.

O segundo parâmetro corresponde à leitura ativa. Este parâmetro repete-se nas demais planilhas uma vez que, como destacado no marco teórico, a realização da leitura ativa é uma característica essencial para o desenvolvimento da leitura social. 
Dentro do critério que avalia a interação com o texto, a leitura ativa é observada pela presença de indicadores vinculados à navegação e à anotação, como a possibilidade de mudar a forma de passar uma página ou seu formato; mudanças tipográficas; busca de palavras ou partes do texto; busca de uma página específica; presença de índice ou tabela de conteúdo; possibilidade de destacar palavras ou partes do texto; de adicionar notas, e de gerar documentos compostos por destaques e anotações realizadas pelo leitor.

\begin{tabular}{|c|c|c|c|}
\hline \multirow{7}{*}{\begin{tabular}{|l|} 
Critério1 \\
Interação com o \\
texto
\end{tabular}} & \multirow{7}{*}{$\begin{array}{l}\text { Parämetros } \\
\text { Paratexto editorial }\end{array}$} & \multicolumn{2}{|l|}{ Indicadores } \\
\hline & & \multirow{4}{*}{$\begin{array}{l}\text { Informações } \\
\text { sobre a obra }\end{array}$} & Título \\
\hline & & & Autor \\
\hline & & & Editora \\
\hline & & & Ano \\
\hline & & \multirow{2}{*}{$\begin{array}{l}\text { Informações } \\
\text { sobre o autor }\end{array}$} & Biografia \\
\hline & & & Bibliografia \\
\hline & \multirow[t]{9}{*}{ Leitura ativa } & \multirow[t]{6}{*}{ Navegação } & Passagem de página \\
\hline & & & Formatos de página \\
\hline & & & $\begin{array}{l}\text { Tipografia (fonte, cores e } \\
\text { tamanho) }\end{array}$ \\
\hline & & & $\begin{array}{l}\text { Busca de palavras ou partes do } \\
\text { texto }\end{array}$ \\
\hline & & & Busca exata de uma página \\
\hline & & & Indice ou tabela de conteúdo \\
\hline & & \multirow[t]{3}{*}{ Anotação } & $\begin{array}{l}\text { Destacar palavras ou partes do } \\
\text { texto }\end{array}$ \\
\hline & & & Adicionar notas \\
\hline & & & $\begin{array}{l}\text { Gerar documento com destaques } \\
\text { e notas }\end{array}$ \\
\hline
\end{tabular}

TABELA 1

Planilha para avaliação de aplicativos - Critério 1: Interação com o texto.

Elaboração própria

\subsubsection{INTERAÇÃO COM OUTROS SISTEMAS}

A segunda planilha (tabela 2), por sua vez, corresponde ao critério da interação que o leitor é capaz de realizar com outros sistemas. Sistemas estes que compóem o ecossistema do livro. Podendo ser sistemas que ampliem a compreensão do texto, sistema de recomendação ou sistemas de compra e acesso. Sendo assim, foram delimitados dois parâmetros, leitura ativa e recomendação.

O parâmetro de leitura ativa é composto por dois tipos de indicadores, relacionados com a busca de significados e a anotação. Evidenciados pela presença de recursos como dicionários, vinculação à Wikipédia, motores de busca ou tradutores, è possibilidade de compartilhar as atividades desenvolvidas durante a leitura por meio de redes sociais, por email ou por vias indicadas pelo próprio aplicativo.

Enquanto isto, dois tipos de indicadores estão vinculados ao parâmetro da recomendação: avaliação e aquisição. $O$ primeiro indicador é fundamentado pela presença de recursos que possibilitem ao leitor avaliar o conteúdo com estrelas ou por meio de texto, como resenhas ou comentários, que permitam compartilhar informações sobre a obra com sistemas de recomendação externos ao aplicativo e que sejam geradas recomendações a partir do livro que for lido. Enquanto isto, o indicador referente à aquisição é fundamentado por recursos como a vinculação com livrarias integradas ao aplicativo ou a outros tipos de meios para aquisição de uma obra com o menor número de ações possíveis fora do aplicativo. 


\begin{tabular}{|c|c|c|c|c|}
\hline \multirow{3}{*}{\begin{tabular}{|l|} 
Critério 2 \\
Interação com \\
outros sistemas
\end{tabular}} & \multirow{11}{*}{ Leitura ativa } & Indicadores & & \\
\hline & & \multirow{5}{*}{$\begin{array}{l}\text { Busca de } \\
\text { significados }\end{array}$} & \multirow[t]{2}{*}{ Dicionários } & Integrado \\
\hline & & & & Livre \\
\hline & & & \multicolumn{2}{|l|}{ Wikipédia } \\
\hline & & & \multirow{2}{*}{\multicolumn{2}{|c|}{$\begin{array}{l}\text { Buscadores } \\
\text { Tradutores }\end{array}$}} \\
\hline & & & & \\
\hline & & \multirow[t]{5}{*}{ Anotação } & \multirow{4}{*}{$\begin{array}{l}\text { Compartilhar } \\
\text { em redes } \\
\text { sociais }\end{array}$} & Facebook \\
\hline & & & & Twitter \\
\hline & & & & Rede própria \\
\hline & & & & Outras \\
\hline & & & \multicolumn{2}{|c|}{ Compartilhar por email } \\
\hline & \multirow[t]{6}{*}{ Recomendação } & \multirow[t]{4}{*}{ Avaliação } & \multicolumn{2}{|c|}{ Avaliar conteúdo com estrelas } \\
\hline & & & \multicolumn{2}{|c|}{$\begin{array}{l}\text { Avaliar conteúdo com texto (resenhas } \\
\text { ou comentários) }\end{array}$} \\
\hline & & & \multicolumn{2}{|c|}{$\begin{array}{l}\text { Compartilhar com sistemas de } \\
\text { recomendação externos }\end{array}$} \\
\hline & & & \multicolumn{2}{|c|}{$\begin{array}{l}\text { Gerar recomendações a partir do livro } \\
\text { lido }\end{array}$} \\
\hline & & \multirow[t]{2}{*}{ Aquisição } & \multicolumn{2}{|c|}{ Livrarias integradas } \\
\hline & & & \multicolumn{2}{|c|}{ Outros acessos } \\
\hline
\end{tabular}

TABELA 2

Planilha para avaliação de aplicativos - Critério 2: Interação com outros sistemas Elaboração própria

\subsubsection{INTERAÇÃO COM OUTROS LEITORES}

Por fim, a terceira planilha (tabela 3) corresponde ao critério relacionado com a capacidade de interação do leitor com outros leitores, impulsionada por recursos presentes no aplicativo de leitura. Para avaliar este critério foram delimitados dois parâmetros. $\mathrm{O}$ de socialização, fundamentado por indicadores relacionados à possibilidade de interagir por meio do próprio texto, em outros espaços, como redes sociais, e por meio do contato com outros leitores dentro do aplicativo. E o da leitura ativa é composto por indicadores de anotação e de aquisição, nos quais são considerados recursos que permitam ao leitor compartilhar ou acessar anotações de outros leitores, e enviar ou receber a obra também de outros leitores.

\begin{tabular}{|c|c|c|c|}
\hline \multirow{6}{*}{$\begin{array}{l}\text { Critério } 3 \\
\text { Interação com } \\
\text { outros leitores }\end{array}$} & Parâmetros & Indicadore: & \\
\hline & \multirow[t]{2}{*}{ Socialização } & \multicolumn{2}{|c|}{ Interação direta (texto) } \\
\hline & & \multicolumn{2}{|c|}{$\begin{array}{l}\text { Interação indireta (fora do texto, redes sociais ou seguir outros } \\
\text { leitores) }\end{array}$} \\
\hline & \multirow[t]{3}{*}{ Leitura Ativa } & \multirow[t]{2}{*}{ Anotação } & Compartilhar anotações \\
\hline & & & Acessar anotações de outros leitores \\
\hline & & Aquisição & Enviar ou receber a obra de outros leitores \\
\hline
\end{tabular}

TABELA 3

Planilha para avaliação de aplicativos - Critério 3: Interação com outros leitores 


\subsection{ANÁliSE dOS APLICATIVOS SELECIONADOS}

Para a avaliação de cada indicador foi seguida a metodologia desenvolvida por Codina (2000) que indica a articulação de três níveis distintos. A superação de cada um dos níveis corresponde a uma escala nominal de qualidade (Codina, 2000), sendo, neste estudo, acrescentado um nível inicial para os casos em que os aplicativos não incluam recursos avaliados em cada indicador.

- Nível 0: Não possui recursos

- Nível 1: Possui recursos porém com limitações

- Nível 2: Possui recursos com desempenho suficiente

- Nível 3: Possui recursos com um desempenho excelente

\subsection{AVAliaÇão dos Benefícios E Limites dos APliCATIVOS PARA A LEITURA SOCIAL}

O processo de análise foi realizado utilizando como base as planilhas descritas anteriormente, no entanto, para uma melhor apresentação dos resultados, estas foram divididas de acordo com os parâmetros estudados. As avaliações foram utilizadas para fundamentar a discussão final sobre os benefícios e limites da prática da leitura social em cada aplicativo analisado.

\section{Resultados e discussão}

A criação dos aplicativos dedicados à leitura representam um considerado avanço no desenvolvimento de práticas de leitura específicas dos ambientes digitais. Este avanço é visível, principalmente, no que concerne à usabilidade, isto é, a proporcionar aos usuários uma série de facilitadores (recursos e ferramentas) para que possam utilizar os dispositivos digitais como instrumentos de estímulo à leitura, incluindo a prática da leitura social, que não sejam percebidos como objetos repletos de distrações ou que impulsionem uma queda na qualidade da leitura.

Entre os aplicativos analisados, apesar de apresentarem características distintas, durante as análises observou-se a existência de diferentes pontos de semelhança. Um deles é a possibilidade de dividi-los em dois gêneros. Estes representam de certa forma o desenvolvimento tecnológico da leitura digital, motivado inicialmente pela criação de dispositivos adequados para este tipo de leitura, e-readers e tablets (Dantas, Mangas-Vega, Goméz-Díaz, \& Cordón-García, 2017). Sendo assim, trata-se de aplicativos ligados a grandes empresas do setor de tecnologia, neste estudo representados por: Google Play Books (Google), Kindle (Amazon), Kobo (Kobo) e iBooks (Apple); e aplicativos criados por desenvolvedores de software independentes interessados em criar inovações tecnológicas capazes de suprir as demandas que surgem cotidianamente, representados por Aldiko, eReader Prestigio, eBoox, Wattpad, Moon+ Reader e Bluefire Reader.

Durante os processos de avaliação verificou-se que as semelhanças extrapolam questões relacionadas aos agentes responsáveis pelo desenvolvimento do aplicativo, se aproximando também às questões relacionadas ao formato, ao acesso, às funções, ou seja, aos recursos que, potencialmente, auxiliam na prática da leitura social, objeto de estudo desta pesquisa. Por esta razão, se decide por apresentar os resultados das avaliações dos aplicativos considerando esse primeiro resultado, isto é, fazendo uma divisão dos aplicativos por grupos (Grupo 1 = grandes empresas; Grupo 2 = desenvolvedores independentes). Considera-se que este tipo de divisão poderá facilitar a verificação de um possível vínculo entre a origem do aplicativo, ligado a grandes grupos ou a empresas de inovação, e os benefícios e possibilidades que um aplicativo pode representar para 
estimular a leitura social. Ou se, em vez disso, a origem é irrelevante e em ambos os casos estão propensos a inserir recursos eficientes para impulsionar esta modalidade de leitura.

Depois de inserido este primeiro resultado nas tabelas utilizadas para avaliar os recursos disponíveis, a primeira análise correspondeu à avaliação do parâmetro denominado de paratexto editorial pertencente ao primeiro critério, relacionado com a interação com o texto.

\begin{tabular}{|c|c|c|c|c|c|c|c|c|c|c|c|}
\hline \multirow{2}{*}{\multicolumn{2}{|c|}{$\begin{array}{l}\text { Critério } 1 \\
\text { Parâmetro: Paratexto } \\
\text { Editorial } \\
\text { Indicadores }\end{array}$}} & \multicolumn{4}{|c|}{$\begin{array}{l}\text { Aplicativos } \\
\text { Grupo } 1\end{array}$} & \multicolumn{6}{|c|}{$\begin{array}{l}\text { Aplicativos } \\
\text { Grupo } 2\end{array}$} \\
\hline & & Pus & (⿻)丨 & $=$ & $m$ & fin: & F & ti & & 0 & \\
\hline \multirow{4}{*}{$\begin{array}{l}\text { Informações } \\
\text { sobre a obra }\end{array}$} & Título & 3 & 3 & 3 & 3 & 3 & 3 & 3 & 3 & 3 & 3 \\
\hline & Autor & 3 & 3 & 3 & 3 & 3 & 3 & 3 & 1 & 3 & 3 \\
\hline & Editora & 3 & 0 & 3 & 3 & 2 & 2 & 0 & 3 & 1 & 3 \\
\hline & Ano & 3 & 0 & 3 & 3 & 3 & 0 & 0 & 1 & 1 & 3 \\
\hline \multirow{2}{*}{$\begin{array}{l}\text { Informações } \\
\text { sobre o autor }\end{array}$} & Biografia & 3 & 0 & 0 & 0 & 0 & 0 & 0 & 1 & 0 & 0 \\
\hline & Bibliografia & 2 & 2 & 2 & 2 & 2 & 2 & 0 & 1 & 0 & 2 \\
\hline \multicolumn{2}{|c|}{ Pontuação total } & 18 & 9 & 15 & 15 & 13 & 10 & 6 & 10 & 12 & 14 \\
\hline
\end{tabular}

TABELA 4

Avaliação do parâmetro paratexto editorial (Critério 1)

Elaboração própria

Como pode ser observado na tabela 4 , todos os aplicativos possuem recursos que auxiliam o leitor a encontrar informações relacionadas ao paratexto editorial. No entanto, a qualidade destes recursos não é completamente satisfatória.

Informações básicas como o ano da publicação e a editora não são facilmente encontradas em grande parte dos aplicativos, simplesmente por não estarem incluídas nas informações principais, como é o caso do Kindle, eReader Prestigio ou eBoox, ou por depender da edição do livro que estiver sendo lido, como foi o caso de Moon+ Reader e Bluefire Reader. Isto é, entende-se que não são informações que os desenvolvedores acreditem que devam ter destaque na seção de informação sobre o livro, independentemente do grupo em que estão incluídos.

Outro resultado que também se destacou foi o que corresponde às informações biográficas do autor. Somente o aplicativo Google Play Books atingiu o nível mais avançado, enquanto a maioria dos aplicativos nem ao menos pontuou.

Os resultados indicam que os aplicativos de leitura parecem não estar aproveitando a oportunidade de poder inserir informações adicionais com relação ao paratexto editorial, principalmente informações que não são facilmente encontradas dentro do contexto impresso, como é o caso da biografia do autor. Ao mesmo tempo, foi possível observar que se prioriza a inserção de indicações de obras da bibliografia do autor ou similares ao livro lido. Este resultado pode estar relacionado com o crescimento de ações do mercado editorial voltadas aos sistemas de recomendação de leitura.

O segundo parâmetro avaliado nesta pesquisa está relacionado com a maior parte dos estudos ligados ao âmbito da leitura ativa dentro do contexto da leitura impressa, ou seja, a possibilidade do leitor de realizar atividades sobre o texto. 


\begin{tabular}{|c|c|c|c|c|c|c|c|c|c|c|c|}
\hline \multirow{2}{*}{\multicolumn{2}{|c|}{$\begin{array}{l}\text { Critério 1 } \\
\text { Parâmetro: Leitura Ativa } \\
\text { Indicadores }\end{array}$}} & \multicolumn{4}{|c|}{$\begin{array}{l}\text { Aplicativos } \\
\text { Grupo1 }\end{array}$} & \multicolumn{6}{|c|}{$\begin{array}{l}\text { Aplicativos } \\
\text { Grupo2 }\end{array}$} \\
\hline & & & & & & & & & & , & \\
\hline \multirow[t]{7}{*}{ Navegação } & $\begin{array}{l}\text { Passagem de } \\
\text { página }\end{array}$ & 1 & 2 & 2 & 2 & 0 & 2 & 3 & 2 & 3 & 2 \\
\hline & $\begin{array}{l}\text { Formatos de } \\
\text { página }\end{array}$ & 2 & 3 & 3 & 2 & 3 & 3 & 3 & 0 & 3 & 3 \\
\hline & $\begin{array}{l}\text { Tipografia (fonte, } \\
\text { cores e tamanho) }\end{array}$ & 2 & 2 & 2 & 2 & 3 & 3 & 2 & 2 & 2 & 3 \\
\hline & $\begin{array}{l}\text { Busca de } \\
\text { palavras ou } \\
\text { partes do texto }\end{array}$ & 3 & 3 & 3 & 3 & 3 & 3 & 3 & 0 & 3 & 3 \\
\hline & $\begin{array}{l}\text { Busca exata de } \\
\text { uma página }\end{array}$ & 3 & 2 & 3 & 3 & 0 & 3 & 0 & 0 & 0 & 0 \\
\hline & $\begin{array}{l}\text { Indice ou tabela } \\
\text { de conteúdo }\end{array}$ & 3 & 3 & 3 & 3 & 0 & 3 & 3 & 3 & 3 & 3 \\
\hline & $\begin{array}{l}\text { Estatística da } \\
\text { leitura }\end{array}$ & 1 & 2 & 3 & 2 & 2 & 1 & 3 & 0 & 3 & 2 \\
\hline \multirow[t]{3}{*}{ Anotação } & $\begin{array}{l}\text { Destacar palavras } \\
\text { ou partes do texto }\end{array}$ & 3 & 3 & 3 & 3 & 3 & 3 & 3 & 0 & 3 & 3 \\
\hline & Adicionar notas & 3 & 3 & 3 & 3 & 1 & 3 & 0 & 3 & 3 & 3 \\
\hline & $\begin{array}{l}\text { Gerar documento } \\
\text { com destaques e } \\
\text { notas }\end{array}$ & 3 & 3 & 0 & 3 & 0 & 0 & 0 & 0 & 3 & 0 \\
\hline \multicolumn{2}{|c|}{ Pontuação total } & 24 & 26 & 25 & 26 & 15 & 24 & 20 & 10 & 26 & 22 \\
\hline
\end{tabular}

TABELA 5

Avaliação do parâmetro leitura ativa (Critério 1)

Elaboração própria

Foi observado que a maior parte dos aplicativos possui recursos que permitem ao leitor realizar atividades básicas e que já eram realizadas durante a leitura de livros impressos, como destacar partes importantes ou escrever informações adicionais. Porém, o que é mais significativo é a inserção de recursos que facilitem as ações que dentro do contexto impresso eram muito mais complexas e, inclusive, poderiam gerar distrações. Como é o caso da busca por páginas especificas, ou por palavras e partes do texto.

O segundo critério avaliado foi o critério interação com outros sistemas, no qual estão incluídos os parâmetros leitura ativa e recomendação (tabela 6).

\begin{tabular}{|c|c|c|c|c|c|c|c|c|c|c|c|}
\hline \multirow{2}{*}{\multicolumn{2}{|c|}{$\begin{array}{c}\text { Critério 2 } \\
\text { Parâmetro: Leitura Ativa } \\
\text { Indicadores }\end{array}$}} & \multicolumn{4}{|c|}{$\begin{array}{l}\text { Aplicativos } \\
\text { Grupo } 1\end{array}$} & \multicolumn{6}{|c|}{$\begin{array}{c}\text { Aplicativos } \\
\text { Grupo } 2\end{array}$} \\
\hline & & 13 & का & $\overline{5}$ & $m$ & fin & m & tat & (III) & 4 & $\pi$ \\
\hline \multirow[t]{5}{*}{$\begin{array}{l}\text { Busca de } \\
\text { significado }\end{array}$} & $\begin{array}{l}\text { Dicionário } \\
\text { Integrado }\end{array}$ & 3 & 3 & 0 & 3 & 0 & 0 & 0 & 0 & 3 & 0 \\
\hline & $\begin{array}{l}\text { Dicionário } \\
\text { Livre }\end{array}$ & 0 & 3 & 3 & 3 & 0 & 1 & 0 & 3 & 3 & 0 \\
\hline & Wikipédia & 3 & 3 & 3 & 0 & 0 & 0 & 0 & 0 & 0 & 0 \\
\hline & Buscadores & 3 & 3 & 3 & 3 & 3 & 0 & 0 & 0 & 3 & 3 \\
\hline & Tradutores & 3 & 3 & 3 & 0 & 1 & 3 & 0 & 3 & 3 & 0 \\
\hline \multirow[t]{5}{*}{ Anotação } & $\begin{array}{l}\text { Compartilhar } \\
\text { Facebook }\end{array}$ & 0 & 3 & 3 & 3 & 3 & 3 & 3 & 3 & 3 & 0 \\
\hline & $\begin{array}{l}\text { Compartilhar } \\
\text { Twitter }\end{array}$ & 0 & 3 & 3 & 3 & 3 & 3 & 3 & 3 & 3 & 0 \\
\hline & $\begin{array}{l}\text { Compartilhar } \\
\text { Rede própria }\end{array}$ & 0 & 3 & 3 & 0 & 0 & 0 & 0 & 3 & 0 & 0 \\
\hline & $\begin{array}{l}\text { Compartilhar } \\
\text { Outras redes }\end{array}$ & 0 & 0 & 3 & 3 & 3 & 3 & 3 & 0 & 3 & 0 \\
\hline & $\begin{array}{l}\text { Compartilhar } \\
\text { Email }\end{array}$ & 3 & 3 & 3 & 3 & 3 & 3 & 3 & 3 & 3 & 0 \\
\hline \multicolumn{2}{|c|}{ Pontuação total } & 15 & 27 & 27 & 21 & 16 & 16 & 12 & 18 & 24 & 3 \\
\hline
\end{tabular}

TABELA 6

Avaliação do parâmetro leitura ativa (Critério 2)

Elaboração própria 
Com relação ao primeiro parâmetro deste critério foi possível observar, mais uma vez, o interesse por parte dos desenvolvedores em incluir atividades que são mais complexas durante a prática da leitura impressa. Isto é, proporcionando ao leitor a possibilidade de acessar diferentes dicionários ou até mesmo traduzir termos sem precisar sair do aplicativo.

Compartilhar a leitura é uma importante ação dentro do contexto da leitura social e é relevante a atenção dada por parte dos aplicativos neste sentido. Inserindo a possibilidade de compartir partes do texto com diferentes redes sociais (por exemplo,Facebook ou Twitter). Mas também utilizando outros aplicativos que estejam instalados no dispositivo móvel (por exemplo, serviços de mensagem), e também por email.

Neste parâmetro (tabela 7), a maior limitação reconhecida é a possibilidade de compartilhar conteúdos dentro de uma rede inserida no aplicativo, identificando-se somente três aplicativos que permitem essa ação: Kobo, Kindle e Wattpad.

\begin{tabular}{|c|c|c|c|c|c|c|c|c|c|c|c|}
\hline \multirow{2}{*}{\multicolumn{2}{|c|}{$\begin{array}{l}\text { Crité rio } 2 \\
\text { Parâmetro: Recomendação } \\
\text { Indicadores }\end{array}$}} & \multicolumn{4}{|c|}{$\begin{array}{c}\text { Aplicativos } \\
\text { Grupo } 1\end{array}$} & \multicolumn{6}{|c|}{$\begin{array}{c}\text { Aplicativos } \\
\text { Grupo } 2\end{array}$} \\
\hline & & & का & $=$ & $\mathrm{m}$ & & & & & & \\
\hline \multirow[t]{4}{*}{ Avaliação } & Avaliar com estrelas & 3 & 3 & 3 & 3 & 0 & 1 & 0 & 1 & 0 & 0 \\
\hline & $\begin{array}{l}\text { Avaliar com texto } \\
\text { (resenhas ou } \\
\text { comentários) }\end{array}$ & 3 & 3 & 3 & 3 & 0 & 0 & 0 & 3 & 0 & 0 \\
\hline & $\begin{array}{l}\text { Compartilhar com } \\
\text { sistemas de } \\
\text { recomendação } \\
\text { externos }\end{array}$ & 0 & 0 & 0 & 0 & 0 & 0 & 0 & 0 & 0 & 0 \\
\hline & $\begin{array}{l}\text { Gerar } \\
\text { recomendações a } \\
\text { partir do livro lido }\end{array}$ & 3 & 3 & 3 & 3 & 0 & 0 & 0 & 3 & 0 & 0 \\
\hline \multirow[t]{2}{*}{ Aquisição } & Livraria integrada & 3 & 3 & 2 & 3 & 3 & 1 & 0 & 2 & 0 & 2 \\
\hline & Outros acessos & 3 & 2 & 1 & 3 & 2 & 2 & 3 & 0 & 3 & 0 \\
\hline \multicolumn{2}{|c|}{ Pontuação total } & 15 & 14 & 12 & 15 & 5 & 4 & 3 & 9 & 3 & 2 \\
\hline
\end{tabular}

TABELA 7

Avaliação do parâmetro recomendação (Critério 2)

Elaboração própria

Como mencionado anteriormente, é notório o interesse por parte dos aplicativos de leitura em gerar recomendações de leitura a seus usuários,o que foi detectado também nesta avaliação.

Grande parte das recomendações é gerada a partir dos dados inseridos pelos leitores, direta ou indiretamente (por exemplo, avaliações das obras ou dados de compras). Como observa-se na tabela 7 , neste quesito existe um maior empenho por parte dos aplicativos ligados às grandes empresas de tecnologia (Grupo 1). Todos os aplicativos pertencentes a este grupo permitem que o leitor insira comentários, resenhas, avaliação com estrelas e, por consequência, possuem sistemas de recomendação altamente visíveis e eficientes. $\mathrm{O}$ mesmo não acontece com os demais aplicativos, que possuem muitas debilidades no que concerne às contribuições que o leitor pode realizar no quesito avaliação/recomendação, reduzindo os dados inseridos aos metadados produzidos pela compra ou pela inserção de um título reconhecido pelo aplicativo.É relevante destacar ainda a falta de conexão direta com outros sistemas de recomendação, como por exemplo, Goodreads, Librarything ou Entrelectores. Plataformas reconhecidas por suas capacidades em auxiliar aos leitores a catalogarem suas bibliotecas, mas também em compartilhar suas leituras e criticas literárias.

O segundo indicador avaliado neste parâmetro (tabela 7) está relacionado com a aquisição de conteúdo. Foi possível observar que a maioria dos aplicativos proporcionam diferentes formas de ter acesso a livros. Seja por meio de lojas integradas diretamente no aplicativo ou pela possibilidade de adquirir os livros em outras plataformas e enviá-los ao aplicativo selecionado. No caso do aplicativo Wattpad, como se trata de um aplicativo voltado para a autopublicação e distribuição gratuita,nesta avaliação indicamos como livraria integrada, mesmo que se trata de um espaço de livros sem custos para o leitor. 
Os únicos aplicativos que não possuem livrarias integradas foram eBoox e Moon+ Reader. De certa forma isto pode ser considerado uma debilidade porque impulsiona a que sempre que o leitor queira buscar um livro deva acessar outras plataformas fora do aplicativo. Ocorrendo o sentido inverso com os aplicativos Wattpad e Bluefire Reader, que limitam os usuários aos sistemas que estão integrados ao aplicativo, ou seja, o leitor só poderá ter acesso a obras que estejam disponíveis nos sistemas integrados.

Finalmente, as últimas avaliações realizadas correspondem a um critério extremamente significante dentro do contexto da leitura social, a interação dos leitores com outros leitores (tabela 8). Não obstante, foi possível considerar que os aplicativos avaliados possuem consideráveis debilidades no que corresponde a impulsionar a socialização entre os leitores.

\begin{tabular}{|c|c|c|c|c|c|c|c|c|c|c|}
\hline $\begin{array}{c}\text { Crité rio } 3 \\
\text { Parâmetro: Socialização }\end{array}$ & \multicolumn{4}{|c|}{$\begin{array}{l}\text { Aplicativos } \\
\text { Grupo } 1\end{array}$} & \multicolumn{6}{|c|}{$\begin{array}{l}\text { Aplicativos } \\
\text { Grupo } 2\end{array}$} \\
\hline Indicadores & ? & & & - & & & & & & \\
\hline Interação direta (t exto) & 0 & 2 & 3 & 0 & 0 & 0 & 0 & 3 & 0 & 0 \\
\hline $\begin{array}{l}\text { Interação indireta (fora do } \\
\text { texto, redes sociais ou seguir } \\
\text { outros leitores) }\end{array}$ & 0 & 0 & 3 & 0 & 0 & 0 & 0 & 3 & 0 & 0 \\
\hline Pontuação total & 0 & 2 & 6 & 0 & 0 & 0 & 0 & 6 & 0 & 0 \\
\hline
\end{tabular}

TABELA 8

Avaliação do parâmetro socialização (Critério 3)

Elaboração própria

Com relação à socialização,apenas três deles permitem a interação entre leitores: Kobo, Kindle e Wattpad. No entanto, no caso do aplicativo Kindle, essa interação dependerá da edição do livro lido, apenas algumas delas tem permitido esse tipo de contato.

É oportuno enfatizar que os recursos que permitem esse tipo de interação representam um considerável avanço, entretanto, muitas vezes se resume a vários leitores que comentam o mesmo trecho do livro, mas que não geram linhas de conversação.

E com relação à interação fora do aplicativo, esse número se reduz a apenas dois, Kobo e Wattpad, os únicos que permitem que os leitores possam interagir entre si tendo como elo o aplicativo de leitura.

\begin{tabular}{|c|c|c|c|c|c|c|c|c|c|c|c|}
\hline \multirow{2}{*}{\multicolumn{2}{|c|}{$\begin{array}{l}\text { Critério } 3 \\
\text { Parâmetro: Leitura Ativa } \\
\text { Indicadores }\end{array}$}} & \multicolumn{4}{|c|}{$\begin{array}{l}\text { Aplic ativos } \\
\text { Grupo } 1\end{array}$} & \multicolumn{6}{|c|}{$\begin{array}{l}\text { Aplicativos } \\
\text { Grupo } 2\end{array}$} \\
\hline & & $n$ & Ex & 5 & $\mathrm{~m}$ & in. & $\theta$ & 4 & 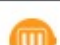 & 4 & \\
\hline \multirow[t]{2}{*}{ Anotação } & $\begin{array}{l}\text { Compartilhar } \\
\text { an otações }\end{array}$ & 3 & 3 & 3 & 0 & 3 & 2 & 3 & 0 & 0 & 0 \\
\hline & $\begin{array}{l}\text { Acessar } \\
\text { anotações de } \\
\text { outros leitores }\end{array}$ & 1 & 2 & 3 & 0 & 0 & 1 & 1 & 0 & 0 & 0 \\
\hline Aquisição & $\begin{array}{l}\text { Enviar ou } \\
\text { receber de } \\
\text { outros leitores }\end{array}$ & 2 & 0 & 2 & 2 & 2 & 2 & 0 & 3 & 3 & 0 \\
\hline \multicolumn{2}{|c|}{ Pontuação total } & 6 & 5 & 8 & 2 & 5 & 5 & 4 & 3 & 3 & 0 \\
\hline
\end{tabular}

TABELA 9.

Avaliação do parâmetro leitura ativa (Critério 3)

Elaboração própria

No tocante aos indicadores apresentados na tabela 9 , se observa um comportamento semelhante à tabela anterior. Isto é, convém destacar a escassa iniciativa por parte dos desenvolvedores em criar espaços de interação entre leitores de uma mesma obra. 
Foi possível detectar também que para a interação se valoriza o uso de ferramentas que possuem objetivos extremamente variados, como é o caso das plataformas Facebook ou Twitter, contudo se desaproveita a oportunidade de impulsionar redes sociais mais voltadas à leitura e aos leitores, uma vez que, como comentado na avaliação referente à recomendação, sequer permitem compartilhar por meio de sistemas especializados em leitura.

Neste sentido, é válido ressaltar a iniciativa dos aplicativos Kobo, Kindle e Wattpad que buscam, justamente, incentivar a criação de redes de leitores ao redor de uma obra literária, permitindo que outros tenham acesso às ações realizadas pelos leitores em uma obra lida utilizando estes aplicativos.

Durante a avaliação relativa à aquisição, também apresentada na tabela 9 , foi possível observar que não existem recursos que motivem o intercâmbio de obras entre leitores nos diferentes aplicativos. No entanto, é importante destacar que, dentro do ecossistema do livro digital, a falta deste tipo de intercâmbio pode ser ocasionada pelo fato de ainda ser objeto de muitas restrições por parte dos sistemas de venda (Mangas-Vega, 2016; Vázquez \& Celaya, 2010).

Considerando que durante esta pesquisa foi avaliado um total de 33 indicadores distintos, utilizando os níveis elaborados por Codina (2000), os aplicativos selecionados poderiam alcançar como pontuação máxima 111 pontos. Como é possível observar na tabela 10, as pontuações foram díspares, com níveis considerados excelentes, como foi o caso do aplicativo Kobo (93 pontos), e outros mais problemáticos, como o aplicativo Bluefire Reader (41 pontos).

\begin{tabular}{|c|c|c|c|c|c|c|c|c|c|c|}
\hline Avaliação final & \multicolumn{4}{|c|}{$\begin{array}{l}\text { Aplicativos } \\
\text { Grupo } 1\end{array}$} & \multicolumn{6}{|c|}{$\begin{array}{c}\text { Aplicativos } \\
\text { Grupo } 2\end{array}$} \\
\hline $\begin{array}{l}\text { Parâmetro } \\
\text { (Critério) }\end{array}$ & 13 & W & nobo & Dan & in & 0 & 4 & (4) & 0 & 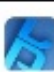 \\
\hline $\begin{array}{l}\text { Paratexto editorial } \\
\text { (Critério 1) }\end{array}$ & 18 & 9 & 15 & 15 & 13 & 10 & 6 & 10 & 12 & 14 \\
\hline $\begin{array}{l}\text { Leitura Ativa } \\
\text { (Critério 1) }\end{array}$ & 24 & 26 & 25 & 26 & 15 & 24 & 20 & 10 & 26 & 22 \\
\hline $\begin{array}{l}\text { Leitura Ativa } \\
\text { (Critério 2) }\end{array}$ & 15 & 27 & 27 & 21 & 16 & 16 & 12 & 18 & 24 & 3 \\
\hline $\begin{array}{l}\text { Recomendação } \\
\text { (Critério 2) }\end{array}$ & 15 & 14 & 12 & 15 & 5 & 4 & 3 & 9 & 3 & 2 \\
\hline $\begin{array}{l}\text { Socialização } \\
\text { (Critério 3) }\end{array}$ & 0 & 2 & 6 & 0 & 0 & 0 & 0 & 6 & 0 & 0 \\
\hline $\begin{array}{l}\text { Leitura Ativa } \\
\text { (Critério 3) }\end{array}$ & 6 & 5 & 8 & 2 & 5 & 5 & 4 & 3 & 3 & 0 \\
\hline Pontuação Final & 78 & 83 & 93 & 79 & 54 & 59 & 45 & 56 & 68 & 41 \\
\hline
\end{tabular}

TABELA 10

Avaliação final

Elaboração própria

É possível constatar que o critério que apresenta uma maior discrepância entre os aplicativos analisados corresponde ao de interação com outros sistemas (critério 2). Este resultado permite afirmar que entre os aplicativos analisados encontramos aqueles que estão centrados unicamente em proporcionar uma ferramenta para ser utilizada para a leitura de livros eletrônicos, sem maiores valores agregados, e, em contrapartida, estão os interessados em proporcionar uma experiência mais ampla de leitura em meios digitais.

No tocante aos limites para a leitura social, sem sombra de dúvidas, a debilidade na interação entre sistemas é um fator de enfraquecimento para o uso de certos aplicativos, uma vez que implicará que para o leitor realizar as atividades previstas nesta modalidade terá que sempre recorrer a diferentes aplicativos, ampliando a possibilidade de dispersão. 
Por outro lado, foi possível avaliar que, com relação à interação entre leitores, a maior parte dos aplicativos tiveram pontuações baixas, o que nos leva a refletir que este campo ainda precisa ser revisado de forma mais aprofundada. Observa-se que a possibilidade de interação entre leitores que utilizem o mesmo aplicativo ou que leiam a mesma obra é limitada a poucos aplicativos e que os principais recursos de compartilhamento da leitura se limitam a plataformas de redes sociais mais generalistas, o que também pode impulsionar a dispersão, ou mesmo não agregar nenhum tipo de experiência social e positiva para a leitura.

Com respeito aos benefícios que a utilização dos aplicativos pode gerar para a prática da leitura social, certamente, os recursos avaliados no critério 1 podem ser considerados os grandes suportes para os leitores. A maior parte dos aplicativos analisados permite que o leitor realize de forma eficiente ações relacionas à leitura ativa sem nenhum tipo de dificuldade.

É importante a presença destes recursos e que funcionem de forma prática porque, como enfatizado no marco teórico deste estudo, realizar ações no texto (por exemplo anotar, sublinhar, etc.) pode auxiliar a que os níveis de compreensão do texto sejam ampliados. Motivando assim a que a leitura social não seja considerada uma modalidade que produza distração, mas sim, que incentive o leitor a utilizar diferentes recursos para compreender melhor a texto lido.

Por fim, apresentamos na tabela seguinte (tabela 11) o ranking dos aplicativos analisados segundo a pontuação pelos recursos presentes que auxiliam ao leitor durante a prática da leitura social. 


\begin{tabular}{|c|c|c|}
\hline Posição & Aplicativo & Pontuação Final \\
\hline $1^{\circ}$ Posição & Kobo & 93 \\
\hline $2^{\circ}$ Posição & $\begin{array}{l}\text { Kindle } \\
\text { Kand }\end{array}$ & 83 \\
\hline $3^{\circ}$ Posição & iBooks & 79 \\
\hline $4^{\circ}$ Posição & $\begin{array}{l}\text { Woogle } \\
\text { Goy Book } \\
\text { Play }\end{array}$ & 78 \\
\hline $5^{\circ}$ Posição & $\begin{array}{l}\text { Moont } \\
\text { Reader }\end{array}$ & 68 \\
\hline $6^{\circ}$ Posição & $\begin{array}{c}\text { O } \\
\text { eReader } \\
\text { Prestigio }\end{array}$ & 59 \\
\hline $7^{\circ}$ Posição & Wattpad & 56 \\
\hline $8^{\circ}$ Posição & Aldiko & 54 \\
\hline $9^{\circ}$ Posição & eBoox & 45 \\
\hline 10 Posição & $\begin{array}{l}\text { Bat } \\
\text { Bluefire } \\
\text { Reader }\end{array}$ & 41 \\
\hline
\end{tabular}

TABELA 11

Ranking de aplicativos para a prática da leitura social Elaboração própria

Analisando o ranking apresentado é possível observar que, efetivamente, o grupo de aplicativos ligados às grandes empresas de tecnologias possuem uma maior capacidade em proporcionar recursos que permitam uma melhor prática da leitura social, respectivamente os aplicativos Kobo, Kindle, iBooks e Google Play Books. Este resultado está diretamente relacionado com a capacidade que estas empresas possuem em criar espaços que permitam a interação de vários sistemas, assim como, nos casos do Kobo e Kindle, por tratarse de empresas que possuem também o interesse em gerar novas experiências de leitura social, para além de, simplesmente, servirem como suporte para a leitura de livros eletrônicos.

\section{Conclusões}

A mudança dos hábitos cotidianos em virtude do intenso contato com diferentes tipos de tecnologias, cada vez mais avançadas, é um fato inegável. Em virtude da evolução do âmbito do livro digital, estas mudanças passaram também a incluir transformações no hábito da leitura. 
Foi observado durante este estudo que, cada vez mais, se criam ferramentas que buscam proporcionar ao leitor novas experiências que não alteraram a qualidade da leitura. Isto é, que siga sendo possível para o leitor atingir os objetivos almejados com a leitura e tenha as mesmas possibilidades de alcançar os níveis de compreensão que se é possível por meio da leitura em ambientes analógicos.

A leitura social possui características próprias e por isto é importante que para realizá-la de forma eficiente o leitor opte por softwares que não apenas sirvam de suporte para a leitura digital, mas que proporcionem os recursos adequados para realizar as atividades incluídas nesta modalidade de leitura.

Os aplicativos móveis são indubitavelmente a melhor resposta para isso. No entanto, foi possível observar que ainda existem alguns pontos que necessitam ser melhor desenvolvidos para que esses espaços supram as necessidades demandadas por um leitor que pretende praticar a leitura social. Destacamos dois pontos em especial: interação entre leitores e informações do paratexto editorial.

Com relação à interação, foi possível observar que a maioria dos aplicativos propõe um compartilhamento limitado a "copiar e colar" as partes interessantes do livro em redes sociais generalizadas, como Facebook e Twitter,não existindo uma integração social forte, que saia da linha da superficialidade, entre os leitores do próprio aplicativo. Essa integração seria capaz de realmente incentivar os leitores a se integrarem ao espaço destinado à leitura e, inclusive, conseguir ampliar seus níveis de leitura por meio da socialização com outras pessoas interessadas pela mesma obra.

Bem como foram observadas muitas limitações relativas às avaliações inseridas pelos leitores. Não ficou completamente claro se este tipo de ação é incentivada com o objetivo de servir para outros leitores se orientarem sobre a qualidade da leitura, ou se simplesmente serão utilizadas como dados para posteriores recomendações automatizadas.

Enquanto isto, com relação às informações do paratexto editorial, ocorre um fenômeno semelhante aos dados de avaliação. Dentro do contexto digital é, teoricamente, mas viável inserir dados mais completos sobre o autor e a sua obra, no entanto, a maior parte dos aplicativos parecem se reduzir a indicar apenas outras leituras passíveis de ser adquiridas nas livrarias integradas.

Sem dúvida o grande desafio enfrentado atualmente pela indústria tecnológica, indústria do livro e todos os demais envolvidos no universo da leitura é criar ambientes adequados para satisfazer as necessidades deste leitor social. Estes ambientes devem valorizar as novas habilidades e necessidades que surgem com a leitura social,estimulando que esta se amplie e não se veja prejudicada por determinadas características do ciberespaço.

É essencial que o leitor não se perca em um mundo de milhões de informações e que as informações inseridas por ele sejam utilizadas de forma eficiente e comprometida. Em um espaço onde a estabilidade ainda não se tornou a prioridade - o que pode ser assumido pela certa frequência na criação de novos aplicativos é relevante criar espaços estáveis para que a leitura social ganhe a mesma estabilidade que a leitura analógica.

\section{REFERÊNCIAS}

Aamri, F. Al, Greuter, S., e Walz, S. P. (2015). Trees of Tales: Designing Playful Interactions to Enhance Reading Experiences. In International Conference onInteractive Technologies and Games (iTAG)(pp. 1-8). IEEE. https: //doi.org/10.1109/iTAG.2015.13

Adler, M. J., e Doren, C. van. (2001). Cómo leer un libro: una guía clásica para mejorar la lectura (2nd ed.). Madrid: Debate S.A.

Codina, L. (2000). Evaluación de recursos digitales en línea. Revista Española de Documentación Cientifica, 23(1), 9-44

Cordón García, J. A., Alonso Arévalo, J., Gómez Díaz, R., e Linder, D. (2013). Social Reading: Platforms, Applications, Clouds and Tags. Oxford: Chandos Publishing.

Dantas, T., Mangas-Vega, A., Goméz-Díaz, R., e Cordón-García, J. A. (2017). Reading research and digital reading research: An overview of the current scientific scenario. Informação \& Sociedade: Estudos, 27(2), 117-131. 
Dantas, T. R. (2017). O leitor social e o desenvolvimento da leitura a partir dos experimentos da Fundación Germán Sánchez Ruipérez. Universidad de Salamanca.

García-Rodríguez, A., e Gómez-Díaz, R. (2015). Las demasiadas aplicaciones: parámetros e indicadores para seleccionar las TopAPP de lectura para niños. Anales de Documentación, 18(2). https://doi.org/10.6018/anale sdoc.18.2.227071

Gómez-Díaz, R. (2012). La evaluación de las aplicaciones de lectura. Em J.-A. Cordón-García, F.,R. CarbajoCascón, Gómez-Díaz, e J. Alonso-Arévalo (Eds.), Libros Electrónicos y Contenidos Digitales En La Sociedad Del Conocimiento: Mercado, Servicios Y Derechos, pp. 291-304. Madrid: Pirámide.

Gómez Díaz, R., García Rodríguez, A., e Cordón García, J. A. (2015). APPrender a leer y escribir: Aplicaciones para el aprendizaje de la lectoescritura. Teoría de La Educación, 16(4), 118-137. https://doi.org/10.14201/eks2015 164118137

Grzeschik, K., Kruppa, Y., Marti, D., e Donner, P. (2011). Reading in 2110 - reading behavior and reading devices: a case study. The Electronic Library, 29(3), 288-302. https://doi.org/10.1108/02640471111141052

Guillén Ríos, J., e Ayuso García, M. (2014). Propuesta teórico-metodológica para evaluar la calidad de bases de datos: aplicación al ámbito de enfermería. Anales de Documentación, 17(2), 1-18. http://dx.doi.org/10.6018/analesd oc.17.2.200691

Kang, M., e Eune, J. (2012). Design Framework for Multimodal Reading Experience in Cross-Platform Computing Devices - Focus on a Digital Bible. Em DRS 2012 conference proceedings. Bangkok: Chulalongkorn University.

Lebert, M. (2008). Project Gutenberg (1971-2008). Disponível em: http://www.gutenberg.org/ebooks/27045

Li, W., e Wu, Y. (2017). Adolescents' social reading: Motivation, behaviour, and their relationship. Electronic Library, 35(2), 246-262. https://doi.org/10.1108/EL-12-2015-0239

Library of Congress. (2000). The Gutenberg Bible. Disponível em: https://www.loc.gov/exhibits/bibles/the-gutenbe rg-bible.html

Mangas-Vega, A. (2016). Los derechos del lector digital: revisión sistemática de la literatura. Métodos de Información, 7(13), 213-245. https://doi.org/10.5557/IIMEI7-N13-213245

Mangen, A. (2008). Hypertext fiction reading: haptics and immersion. Journal of Research in Reading, 31(4), 404-419. https://doi.org/10.1111/j.1467-9817.2008.00380.x

Orosa, B. G., e García, X. L. (2016). Digital books rely on social reading to find new success proposals and formats. Anales de Documentación, 19(2). https://doi.org/10.6018/analesdoc.19.2.246351

Palilonis, J., e Bolchini, D. (2015). Active reading behaviors in tablet-based learning. Journal of Educational Multimedia and Hypermedia, 24(3), 235-261.

Patón-Rodríguez, N., e Gómez-Díaz, R. (2016). La evaluación de las aplicaciones de lectura web: un paso más en el proceso de editorialización de la web. Alabe, 14, 1-23. https://doi.org/10.15645/Alabe2016.14.5

Pearson, J., Buchanan, G., Thimbleby, H., e Jones, M. (2012). The Digital Reading Desk: A lightweight approach to digital note-taking. Interacting with Computers, 24(5),327-338. https://doi.org/10.1016/j.intcom.2012.03.001

Pilcher, J. (2016). Mobile Apps for Educational Purposes. Journal for Nurses in Professional Development, 32(5), E15-7. https://doi.org/10.1097/NND.0000000000000287

Richardson Jr, J. V, e Mahmood, K. (2012). eBook readers: user satisfaction and usability issues. Library Hi Tech, 30(1), 170-185. https://doi.org/10.1108/07378831211213283

Romero, N. (2012). Social reading and the creation of customer loyalty clubs or communities to improve communication with our users and reduce costs in marketing and advertising. Bottom Line, 25(2). https://doi .org/10.1108/08880451211256388

Rowberry, S. P. (2016). Commonplacing the public domain: Reading the classics socially on the Kindle. Language and Literature, 25(3). https://doi.org/10.1177/0963947016652782

Sam-Anlas, C. A., e Stable-Rodríguez, Y. (2016). Evaluación de la accesibilidad web de los portales del Estado en Perú. Revista Española de Documentación Cientifica, 39(1), e120. https://doi.org/10.3989/redc.2016.1.1213 
Schrock, K. (2016). Critical evaluation of mobile apps. Disponível em: http://www.ipads4teaching.net/critical-eval-o f-apps.html/

Tveit, Å. K., e Mangen, A. (2014). A joker in the class: Teenage readers' attitudes and preferences to reading on different devices. Library \& Information Science Research, 36(3-4), 179-184. https://doi.org/10.1016/j.lisr.20 14.08.001

Vázquez, J. A., \& Celaya, J. (2010). Derechos de los lectores de libros digitales. Disponível em http://www.dosdoce.com /2010/05/30/derechos-de-los-lectores-de-libros-digitales/

Vlieghe, J., Muls, J., e Rutten, K. (2016). Everybody reads: Reader engagement with literature in social media environments. Poetics, 54. https://doi.org/10.1016/j.poetic.2015.09.001 